\title{
Low-Volume PEEK Gas Cell for BTEX Detection using Portable Deep-UV Absorption Spectrophotometry
}

\author{
Sulaiman Khan ${ }^{1,2,3}$, David Newport ${ }^{1}$ and Stéphane Le Calvé ${ }^{2,3^{*}}$ \\ ${ }^{1}$ School of Engineering, Bernal Institute, University of Limerick, Limerick, Ireland. \\ sulaiman.khan@ul.ie, david.newport@ul.ie \\ ${ }^{2}$ Université de Strasbourg, CNRS, ICPEES UMR 7515, F-67000 Strasbourg, France. \\ slecalve@unistra.fr. \\ ${ }^{3}$ In'Air Solutions, Strasbourg France. \\ *corresponding author (slecalve@ unistra.fr)
}

\begin{abstract}
Monitoring of indoor air quality by detecting individual airborne pollutant is essential for maintaining a healthy indoor environment. UV absorption spectrophotometry coupled with gas chromatography offers a reliable, self-referenced and non-destructive technique for the identification and detection of gas molecules. This paper presents a deep-UV absorption spectrophotometer coupled with a micro gas-chromatography $(\mu \mathrm{GC})$ for the detection of benzene, toluene, ethylbenzene and xylenes (BTEX). The spectrophotometer was developed using a low-volume gas cell made of PolyEther Ether Ketone polymer (PEEK) tube, connected with a portable deep-UV LED and photomultiplier tube. The performance of the detection unit was evaluated with different concentrations of toluene (5-100 $\mathrm{ppm}$ ) in nitrogen and a sensitivity of $107.1 \mu \mathrm{AU} / \mathrm{ppm}$ with a limit of detection of $1.41 \mathrm{ppm}$ was obtained. The detector was incorporated into a micro gas-chromatography setup and high quality chromatograms, having all the peaks separated with good repeatability were obtained for BTEX molecules. The deepUV absorption spectrophotometer developed demonstrates low-volume, low-cost, and ease of development and integration. Whilst demonstrated for BTEX in a nitrogen carrier gas, the spectrometer has the potential to be applied to chromatographic analysis of different analytes in gas or liquid media.
\end{abstract}

Key words:

Gas sensors, absorption spectrophotometry, photomultiplier tubes, UV LEDs, PEEK gas cell, micro Gas chromatography 


\section{Introduction}

Currently, there is considerable interest in detecting and monitoring of different airborne pollutants in indoor spaces. Indoor air can contain harmful pollutants, like volatile organic compounds (VOCs) [1], [2]. Among VOCs, Benzene, Toluene, Ethylbenzene and Xylene (BTEX) are considered to be a hazardous pollutant, and long-term exposure can harm human health. BTEX belong to the group of aromatic hydrocarbons and can be found in indoor and outdoor environments. Its concentration is relatively high in indoor spaces [3] and its primary sources of generation are cigarette smoking, combustion, coal burning, cleaning products, floor adhesives, paint and wood panelling [4]-[5]. Exposure to BTEX is related to sick building syndrome [6]. Among BTEX, Benzene is highly toxic and is recognised as carcinogenic by the International Agency for Research on Cancer [7]. Benzene can provoke narcosis, headache, dizziness, drowsiness, confusion, tremor and loss of consciousness in case of acute occupational exposure [8][9]. Exposure to toluene is linked with central nervous system, liver, kidney and skin-related issues [10]. Xylenes at a low level can cause fatigue, tremor, respiratory, kidney and cardiovascular-related problems [11]. Due to their human toxicity, stringent regulations about the exposure limits to BTEX molecules are established. For instance, the US National Institute of Occupational Safety and Health (NIOSH) have defined an exposure limit of 100 ppm for air-borne toluene for 8-10 time weighted-average [12].

In order to detect BTEX, a sensitive, portable, low-cost gas sensor with ease of integration with other devices, for instance, gas chromatography is needed. Different types of gas sensors have been developed for BTEX detection, such as electrochemical sensors [13], metal oxide semiconductor (MOS) sensors [14], photo-ionization detectors(PID) [15], piezoelectric sensors [16] and optical gas sensors [17][18][19]. MOS sensors usually need high temperature for operation and are sensitive to external humidity. Electrochemical sensors suffer from ageing but have zero drift. PIDs have a major limitation in terms of selectivity, cost, and complex electronics. Piezoelectric-based sensors experience significant noise and also interference from humidity and temperature. On the other hand, optical gas sensors have high sensitivity with minimal drift issues and a rapid time response. Absorption based optical sensors (i.e. spectrophotometer) are considered to be a sensitive and selective technique with minimal drifts or interference issues. In such sensors, the transduction principle is based on direct measurement of the molecule's physical property (i.e. absorbance at a specific wavelength), which is often called as fringerprints of a molecule. Absorption spectrophotometry is a self-reference, non-destructive and reliable approach for detection of gas molecules. It has a quick time response with a low cross-response to other gases, and the target gas molecules are detected without changing its molecular nature [20][21][22]. These features make absorption spectrophotometry an attractive option for gas monitoring in indoor spaces .

BTEX species absorb strongly in deep-UV range ( wavelength $\lambda, 240-275 \mathrm{~nm}$ ) which can be exploited to develop a deep-UV absorption spectrophotometer for BTEX detection. The common 
spectrophotometric instrumentation developed for visible and infrared radiation are not suitable for deep-UV applications due to the high-energy $(6.89 \mathrm{eV}-4.43 \mathrm{eV}$ for $\lambda=180 \mathrm{~nm}-280 \mathrm{~nm})$ of deep-UV radiation. However, recent advancements in UV optoelectronics and semiconductor research have led to the development of efficient and portable emission sources and photodetectors such as LEDs and photomultiplier tubes (PMT) respectively. LEDs provide a portable, spatially stable with lower intensity drifts, quasi-monochromatic, low-powered, cost-effective UV emission source compared to the conventional UV emission sources, for instance, mercury lamp. PMTs offer a sensitive and portable UV photodetector with a high surface area and fast time response even for a low-intensity of light. In addition to the UV emission source and UV photodetector, a low volume gas cell is also needed to realise a portable spectrophotometer with high sensitivity. A low volume gas cell is critical in gas chromatography for the detection of low concentrations of analytes. Gas chromatographs are usually employed in conjunction with detectors such as mass spectrometer or FID which limit its portability due to their size and weight.

A deep UV absorption spectrophotometer coupled with gas chromatography offers a sensitive, reliable and portable solution for the identification and detection of BTEX molecules. Previous research in the application of deep-UV absorption spectrophotometry for BTEX detection was primarily based on improving the sensitivity of the sensor. Eckhardth et al. [23], [24] demonstrated a deep-UV detector using an aluminium-coated HCW (length, $1 \mathrm{~m}$ and diameter, $1 \mathrm{~mm}$ ) using a lamp as a UV source (30W deuterium) and CCD based spectrometer as a UV photodetector. The setup was tested with a gas chromatography column (GC) for detection of aromatic hydrocarbons such as ethylbenzene and bromobenzene. Its' major drawbacks are the size of the setup (emission source and detector) and high volume of the gas cell $(785 \mu \mathrm{L})$. Recently Bui et al. [25] developed a deep-UV absorbance spectrophotometer with a good sensitivity $(152 \mu \mathrm{AU} / \mathrm{ppm}$; limit of detection of $0.66 \mathrm{ppm}$ for toluene) using a deep-UV LED ( $\left.\lambda_{\text {peak }}, 260 \mathrm{~nm}\right)$ and photodiodes for the direct detection of BTEX molecules. An aluminium tube (length, $40 \mathrm{~cm}$ and inner diameter, $2 \mathrm{~mm}$ ) with inner volume $(1256 \mu \mathrm{l})$ was used as a gas cell. The higher inner volume limits its applicability for $\mu \mathrm{GC}$ applications. Recently, low-volume fluidics components have been developed using PolyEther Ether Ketone (PEEK), which has excellent chemical resistance, high melting temperature $\left(343^{\circ} \mathrm{C}\right)$ and good mechanical properties [26]. It is a thermoplastic polymer belongs to a polyaryleetherketone family. It is machinable which give the flexibility to design it according to the desired applications.

This work reports the development of a low volume gas cell integrated with deep-UV LED and PMT for micro gas chromatography of BTEX using deep-UV absorption spectrophotometry. We have employed a PEEK based tubes with connectors as a gas cell owing to its low volume, chemical and optical properties. To our knowledge, the application of low-volume gas cell coupled with LED and photodetector for $\mu \mathrm{GC}$ applications has not been reported. 


\section{Material and Methods}

A deep-UV LED (Mightex System, USA) with peak wavelength, $260 \mathrm{~nm}$ and power range, 45-80 $\mu \mathrm{W}$ was used as a source. The LED was equipped with a passive heat exchanger to minimise the temperature induced noise and shift. For stable UV signals, a constant stable current (0-100 mA) was supplied to the LED. A low-powered photomultiplier tube (H10722-210, Hamamatsu Japan) was employed as a UV-photodetector with an operating wavelength range of 230-700 nm. PEEK tube (inner diameter $500 \mu \mathrm{m}$, IDEX Health \& Science,USA ) was used as a gas cell with custom-made ferrules and nuts for a leakage-free connection. A PEEK T-Assembly (inner diameter $1 \mathrm{~mm}$, IDEX Health \& Science,USA ) was micromachined to achieve a leak-free sealing and optical alignment with optical fibre. For optical connection, custom made connectors, i.e. nuts were micromachined and fitted onto the SMA connector of optical fibre (QP400-Solarization resistant, 200-1100 nm, Ocean optics) with orings to ensure sealing. The schematic of the spectrophotometric setup is given in Figure 1. The sealing of the assembly was tested with nitrogen by using a mass-flow controller at the inlet and a mass-flow meter at the outlet of the setup, and gas leakages less than $2 \%$ were observed. All the components were assembled to minimise the dead volume in the setup. The gas cell was placed in a 3D-printed holder, fixed on an optical breadboard (Thorlabs USA) to keep the fibre straight and to avoid any mechanically induced noise. The assembly was also optically and thermally insulated to avoid any stray light, and thermal-induced fluctuations A 3D-printed holders and connectors (Acrylonitrile Butadiene StyreneABS) were used to aligned and fixed all the components on the breadboard.

For direct injection tests, the concentration of the toluene mixture was varied by diluting the flow from the toluene cylinder (100 ppm $\pm 2 \%$, Air Products, France) with $\mathrm{N}_{2}\left(99.99 \% \mathrm{~N}_{2}\right.$, Air Products, France) at different flow ratios using mass flow controllers ( F-201CV, Bronkhorst, Netherland) with a full-scale range of $20 \mathrm{~mL} / \mathrm{min} \pm 0.5 \%$ and $10 \mathrm{~mL} / \mathrm{min} \pm 0.5 \%$, respectively. The setup for the generation of different concentration is given in Figure S1.

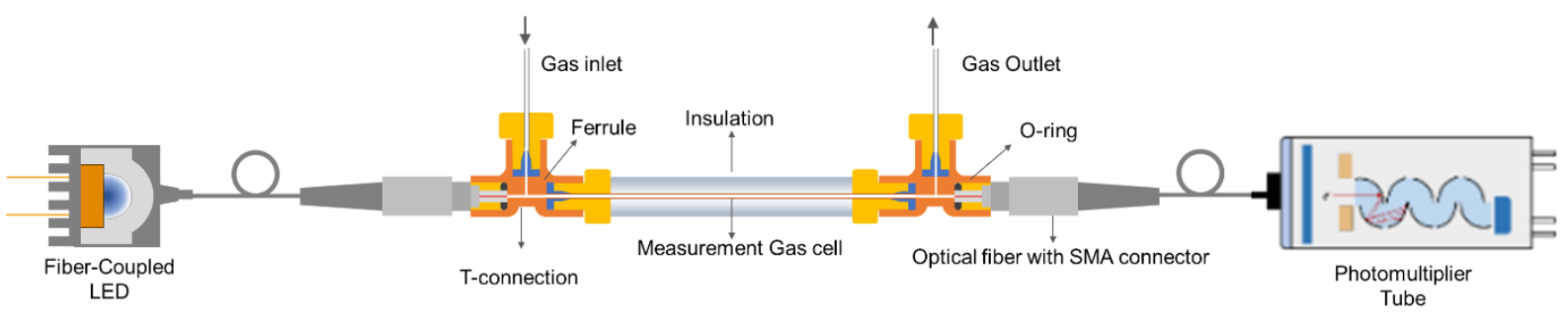

Figure 1. Deep-UV Absorption spectrophotometer with low volume gas cell coupled with LED and PMT.

For gas chromatography (GC), a chromatography setup was employed as given in Figure 2. A similar prototype was investigated and optimized for BTEX detection by our research group [27] [28]. A PDMS based column with length of $20 \mathrm{~m}$ (Inner diameter $180 \mu \mathrm{m}, \mathrm{RXi}-624 S i l \mathrm{MS}$ ) coiled into a 
small circle with diameter of $7.5 \mathrm{~cm}$ placed inside an aluminium plate $(10 \mathrm{~cm} \times 10 \mathrm{~cm} \times 1 \mathrm{~cm})$ was used for separation. The column was heated with an electrical resistor with a thermocouple placed under the column. The temperature was regulated via a controller (CAL3300 Farnel, France). The column was thermally insulated and the analysis was perferomed at a constant temperature of $60^{\circ} \mathrm{C}$. The $\mu \mathrm{GC}$ column was coupled with a solenoid 6-port value (MTV-6LL-N32UF-1, Takasago Japan) for diverting the flow direction during different phases of chromatrography. The sampling loop of volume $200 \mu \mathrm{L}$ (PEEK Interchim France) was connected with the 6-port value. The data was acquired from the PMT using a custom-made software at a sampling rate of $1.0 \mathrm{Sample} / \mathrm{sec}$. The data post-processing was performed using Matlab and Spectragryph [29]. A photoionization detector (PID, Baseline-Mocon USA) was installed at the exit of the gas cell for validation of the signal. For spectral analysis, the emission spectra of the LED was recorded using a mini-spectrometer (Hamamatsu C10082CH, Japan).

For GC analysis, a BTEX cylinder (Air Products, France) with a total concentration of $60 \mathrm{ppm}$, with each molecule at a concentration of $10 \mathrm{ppm} \pm 2 \%$ was used as a source of BTEX mixture. Different concentrations of BTEX mixture (i.e. $10 \mathrm{ppm}, 5 \mathrm{ppm}$ and $2.5 \mathrm{ppm}$ ) were generated using the dilution setup as shown in Figure S1 (Supplementary file). The supply of the carrier gas is maintained inside the gas chromatograph through a pressure controller (IQP600, 0-10bar $\pm 5 \%$ Bronkhorst, Netherland).

(a)

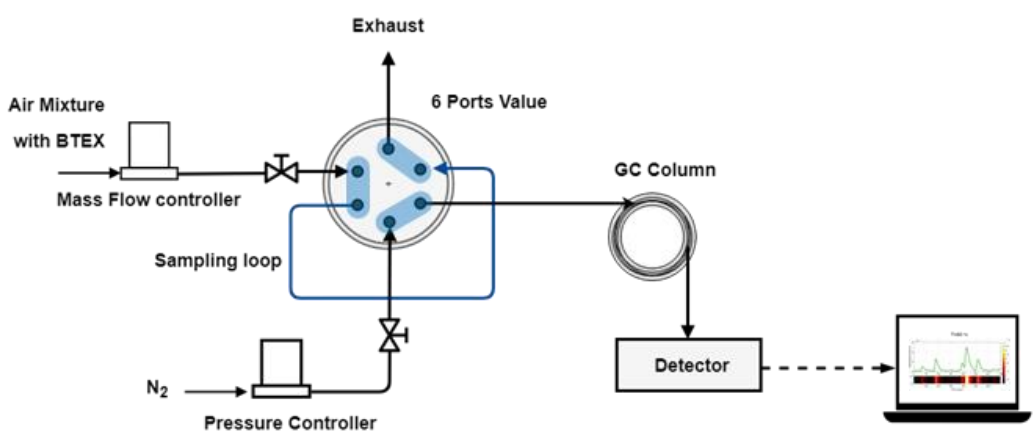

(b)

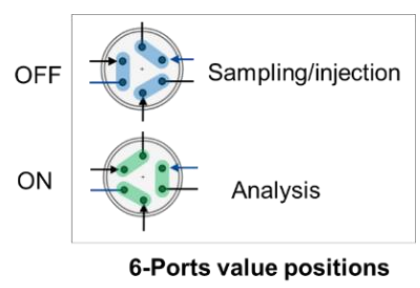

Figure 2. (a) Schematic of $\mu G C$ setup with deep-UV absorption spectrophotometer for BTEX detection. (b) Value position for sampling and analysis mode of $G C$.

\section{Results and Discussion}

In order to investigate the prototype for absorbance measurement of BTEX mixture, the emission spectrum of the LED was compared with the absorption spectra for BTEX molecules. The emission spectra of the LED was measured with a mini-spectrometer and mapped on the absorption spectra of BTEX molecules, as shown in Figure 3(a). The absorption data of benzene, toluene and xylene were obtained from high-resolution transmission molecular absorption database (HITRAN) [30]. The LED's spectrum matches significantly with the absorption spectrum of BTEX molecules, which implies that the LED can detect the peak absorbance of BTEX molecules in the wavelength range of $245 \mathrm{~nm}$ to $275 \mathrm{~nm}$. The LED shows emission peak centered at $260 \mathrm{~nm}$ while benzene, toluene and xylene(p-) have maximum peak absorbance at $253 \mathrm{~nm}\left(7.73 \times 10^{-18} \mathrm{~cm}^{2}\right.$-molecule $\left.{ }^{-1}\right), 266.8 \mathrm{~nm}\left(1.21 \times 10^{-18} \mathrm{~cm}^{2}\right.$ - 
161

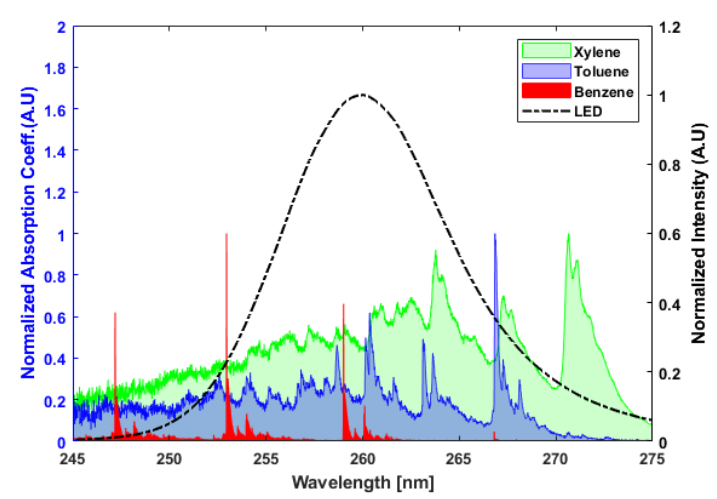

molecule $\left.{ }^{-1}\right)$ and $270.6 \mathrm{~nm}\left(1.15 \times 10^{-18} \mathrm{~cm}^{2}-\right.$ molecule $\left.^{-1}\right)$, respectively. For comparing the integrated absorbance of each molecule in the wavelength region corresponding to the LED emission, area integrals of BTEX were calculated by using area under the absorbance spectrum for a wavelength range 245-275 nm. Xylenes show high average absorbance, followed by toluene as shown in Figure 3(b). On the other hand, benzene has the lowest average absorbance among the BTEX molecules (4 times lower than xylenes).

(b)

(a)

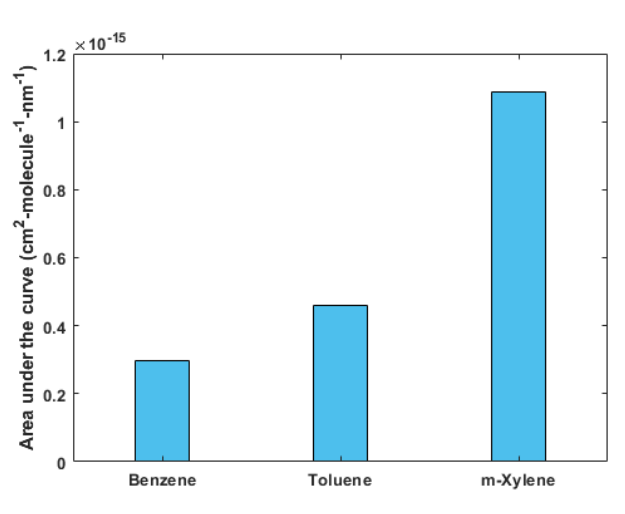

167

168

169

170

171

172

Figure 3. (a) LEDs emission spectrum overlaid with the absorption spectrum of benzene, toluene and xylene(-m). Data was obtained from HITRAN and then plotted according to the scale. Each spectrum is normalized by its maximum peak value. (b) The area integral calculated for a wavelength range (245nm to $275 \mathrm{~nm})$.

Before testing the prototype with BTEX using the $\mu \mathrm{GC}$, experiments were performed using different concentrations of a gaseous toluene-nitrogen mixture. PEEK optical gas cells with an optical path length of $7.5 \mathrm{~cm}, 30 \mathrm{~cm}$ and $50 \mathrm{~cm}$ were designed and coupled into the setup as shown in Figure 1. The dimensions and the corresponding total volume for each cell are summarized in Table 1. For a cell of $50 \mathrm{~cm}$, the total volume was $133.52 \mu \mathrm{L}$ by taking into account the volume of each T-assembly at the two ends of the gas cell. For the configuration of $30 \mathrm{~cm}$, the two T-assemblies contribute significantly $(37 \%)$ to the total volume of the cell. Indeed, the T-assemblies were designed with a relatively higher inner diameter $(1.5 \mathrm{~mm})$ than the gas cell's inner diameter $(0.5 \mathrm{~mm})$ to avoid the optical misalignment and gas leakage.

Table 1. The total inner volume of the gas cell assembly with different length of gas cell.

\begin{tabular}{|c|c|c|c|c|c|c|c|}
\hline \multirow{2}{*}{$\begin{array}{c}\text { Cell. } \\
\text { No }\end{array}$} & $\begin{array}{c}\text { Length } \\
(\mathrm{cm})\end{array}$ & $\begin{array}{c}\text { Inner } \\
\text { diameter } \\
(\mathrm{mm})\end{array}$ & $\begin{array}{c}\text { Volume } \\
(\mu \mathrm{L})\end{array}$ & $\begin{array}{c}\text { Length } \\
(\mathrm{cm})\end{array}$ & $\begin{array}{c}\text { Inner } \\
\text { diameter } \\
(\mathrm{mm})\end{array}$ & $\begin{array}{c}\text { Volume } \\
(\mu \mathrm{L})\end{array}$ & $\begin{array}{c}\text { Total } \\
\text { Volume } \\
(\mu \mathrm{L})\end{array}$ \\
\hline 1 & 7.50 & 0.50 & 14.73 & 2.0 & 1.50 & 35.34 & 50.07 \\
\hline 2 & 30.0 & 0.50 & 58.90 & 2.0 & 1.50 & 35.34 & 94.25 \\
\hline 3 & 50.0 & 0.50 & 98.17 & 2.0 & 1.50 & 35.34 & 133.52 \\
\hline
\end{tabular}


Different concentrations of toluene in $\mathrm{N}_{2}$ were then injected into the gas cell, and the transmitted intensity of UV through the gas cell was recorded using the PMT. The PMT tube was employed as a UV photodetector due to its high sensitivity, higher surface area and compensation for the optical losses associated with the PEEK gas cell. The absorbance was calculated using the Beer-Lambert law as expressed as,

$$
A=\log \frac{I_{N_{2}}-I_{\text {dark }}}{I_{\text {Tol }}-I_{\text {dark }}}
$$

Where $A$ is absorbance, $I_{d a r k}$ is the intensity measured when LED is OFF, while $I_{N 2}$ and $I_{T o l}$ represent the average intensity, after stabilisation, measured for the nitrogen and toluene mixture injections, respectively. For instance, Figure 4 shows the intensity signal recorded with the UV absorption spectrophotometer for $30 \mathrm{ppm}$ of toluene-nitrogen mixture, where $\mathrm{N}_{2}$ and toluene-nitrogen mixture were injected alternatively by closing one two-way valve and opening another. Once the reference intensity obtained with pure nitrogen, the transmitted intensity decreases when the toluene mixture is injected due to absoprtion of UV by the toluene gas molecules. In addition, it was observed that the reference transmitted intensity decreases with time, suggesting a slight decrease in light intensity with time. This baseline drift could also be attributed to the thermal fluctuations and electronic setup related noise. A narrow peak was observed at the instant of injection of toluene mixture. Note that no such narrow peaks were observed when the detection cell was coupled to the $\mu \mathrm{GC}$ (Figure 6), where the gas flow is continuous without any value opening or closing. Therefore, the gas flow dynamics inside the microtube at the instant of valve opening is a potential reason for this effect. The gas cell of length $7.5 \mathrm{~cm}, 30 \mathrm{~cm}$ and $50 \mathrm{~cm}$ were tested for different toluene concentrations, and good linearities $\left(\mathrm{R}^{2} \sim 0.99\right)$ were obtained for all the lengths of the gas cell as shown in Figure 5. This indicates a good agreement to the Beer-Lambert law and minimal influence of dark current and stray light on the PMT. The sensitivities of $42.0 \mu \mathrm{A} . \mathrm{U} / \mathrm{ppm}, 107.1 \mu \mathrm{A} . \mathrm{U} / \mathrm{ppm}$ and $194.4 \mu \mathrm{A} . \mathrm{U} / \mathrm{ppm}$ were obtained for a gas cell of $7.5 \mathrm{~cm}, 30 \mathrm{~cm}$ and $50 \mathrm{~cm}$, respectively. The sensitivity obtained for the gas cell of $30 \mathrm{~cm}$ with inner volume of 94.25 $\mu \mathrm{L}$ is comparable with the sensitivity reported by Bui et al. [25] for toluene using aluminum gas cell with optical path length and inner volume of $40 \mathrm{~cm}$ and $1256 \mu \mathrm{L}$ respectively. A limit of detection of $1.41 \mathrm{ppm}$ was estimated for the gas cell of $50 \mathrm{~cm}$ using a concentration corresponding to a signal equal to 3 times the baseline noise. 


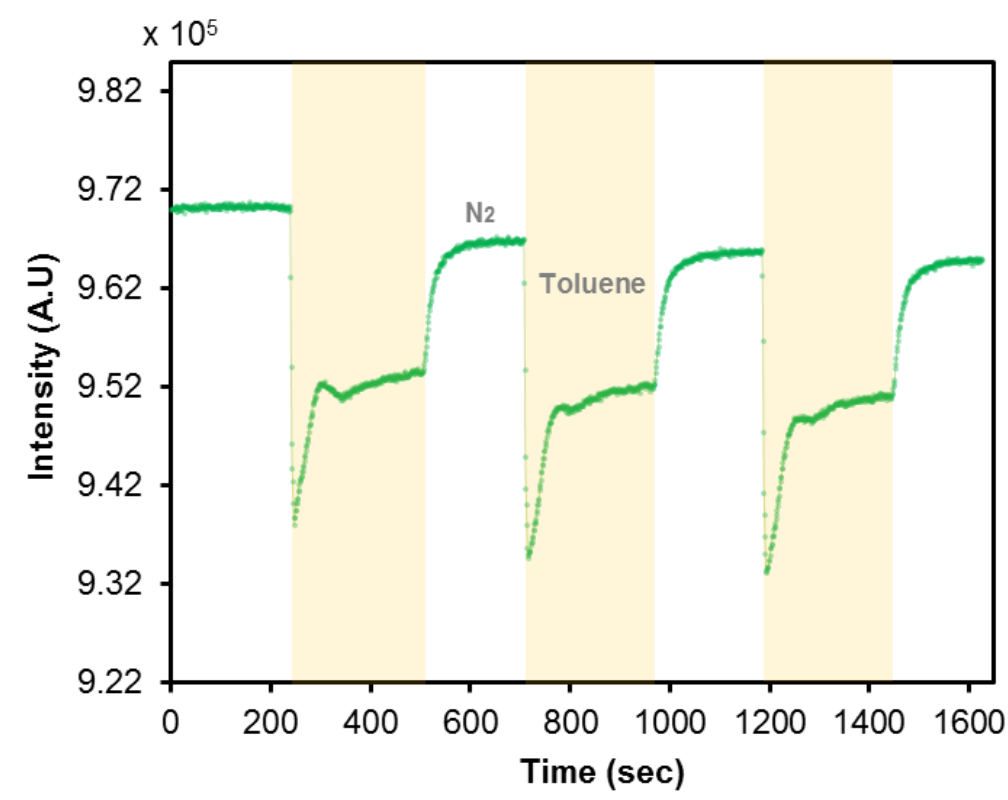

Figure 4. Variation of intensity with pure nitrogen and 30 ppm alternate toluene injections for a gas cell of $50 \mathrm{~cm}$. The 211 vertical bars represent the injection duration of toluene mixture.

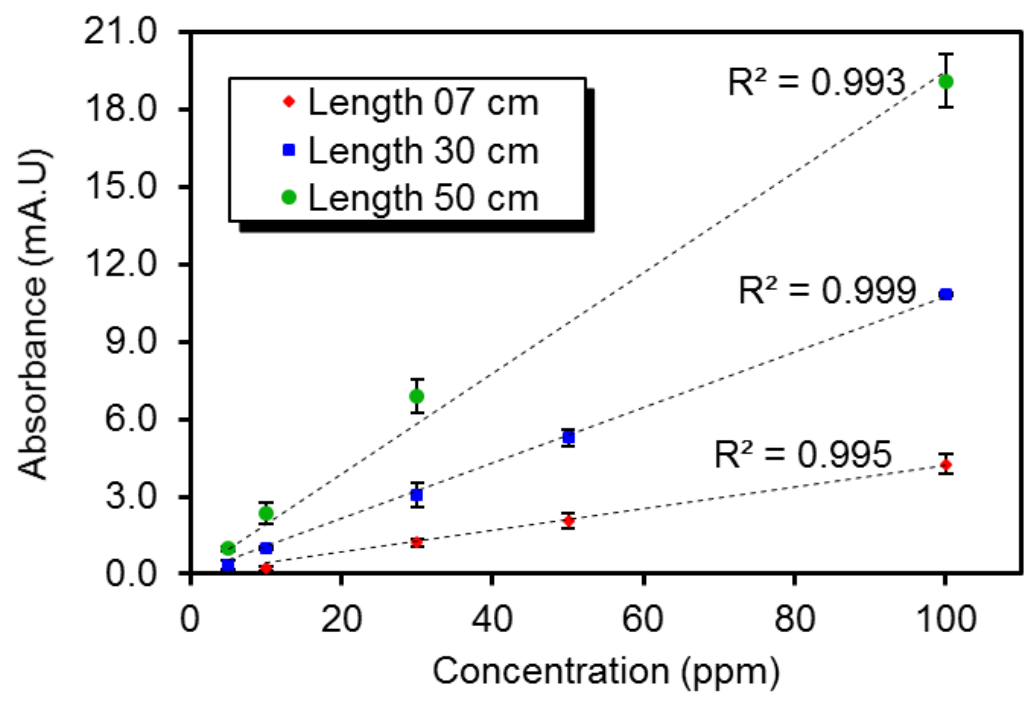

Figure 5. Calibration curve for gaseous toluene concentration ranging between 5 and 100 ppm using different size of PEEK cell.

b. Detection of BTEX mixture in $N_{2}$ with $\mu G C$

The UV absorption spectrophotometer was extended for separation and detection of a BTEX mixture using a $\mu \mathrm{GC}$. A $50 \mathrm{~cm}$ PEEK gas cell was employed as a gas cell by taking into account the lower absorbances of benzene and ethylbenzene molecules. The dimensions and the total volume of the gas cell are given in Table 1. The sampling, separation and detection of BTEX mixture were carried out in two modes: i.e. sampling mode and analysis mode. In the sampling mode, a mass flow controller was used to inject different concentration of BTEX into the sampling loop for a time of $15 \mathrm{sec}$. The transition from the sampling mode to the analysis mode was performed by using a solenoid 6-port valve as shown 
in Figure 2. The separation was carried out by a microcolumn at $60^{\circ} \mathrm{C}$ with a controlled heating process. During the sampling mode, the 6-port valve was OFF, allowing the BTEX mixture to pass through the sampling loop at a controlled flow rate of $2.0 \mathrm{~mL} / \mathrm{min}$. The carrier gas $\left(\mathrm{N}_{2}\right)$ was passing through the column and detector at a flow rate around $2.45 \mathrm{~mL} / \mathrm{min}$ in the sampling mode as shown Figure 2. In the analysis mode, the BTEX molecules were separated in the $\mu$ GC column followed by UV absorption by means of LED and PMT. For this, the valve was turned ON which diverted the carrier gas $\left(\mathrm{N}_{2}\right)$ flow direction from the exhaust into the sampling loop as represented in the inset of Figure 2. The sampled mixture of BTEX was then pushed into the GC column and then into the UV detection cell. The analysis and detection mode run for a typical time period of about $900 \mathrm{sec}$ to obtain a complete chromatogram for the BTEX mixture.
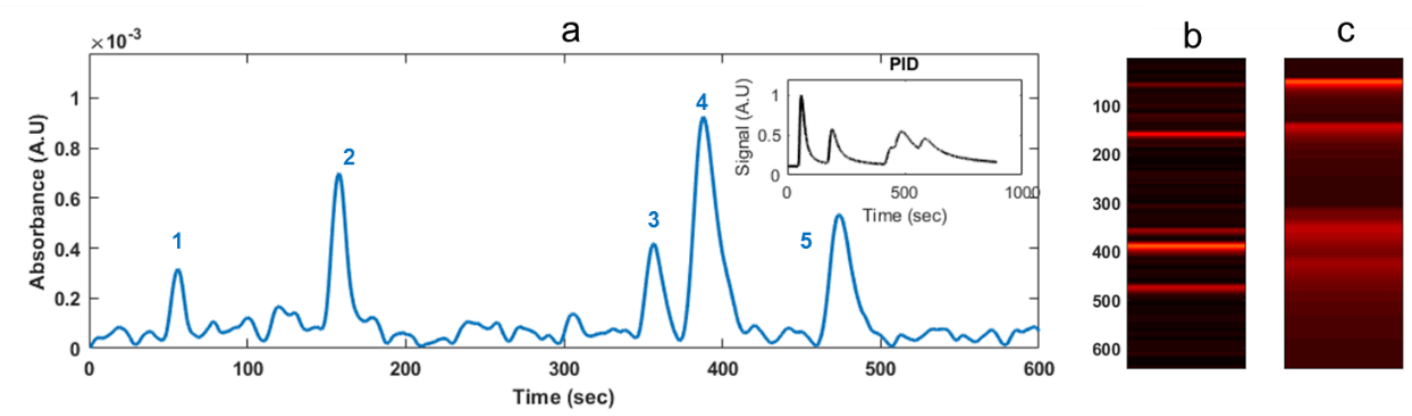

Figure 6. (a) Chromatogram of 10 ppm BTEX mixture obtained using $50 \mathrm{~cm}$ PEEK cell at $\mu G C$ column temperature of $60^{\circ} \mathrm{c}$. The inset plot is the signal obtained from PID. The order of peaks is (1) benzene, (2) toluene, (3)ethylbenzene, (4) $m+p$ xylenes (4) and (5) o-xylene. Representation of chromatograms from (b) UV absorption spectrophotometer, and (c) PID for comparison of separation time.

A 60 ppm BTEX mixture, with each component of concentration 10 ppm was injected into the $\mu \mathrm{CC}$ coupled to the PEEK gas cell equipped with the UV absorption spectrophotometer. The chromatogram obtained is shown in Figure 6, where each peak represents individual BTEX species, except for the mand p-xylenes which are coeluted. The UV absorption detection exhibits the highest and lowest sensitivity for xylenes $(m+p)$ and benzene respectively, while its sensitivity is more moderate for toluene, according to the spectral data as discussed above. The sequence of the peaks detected shows adherence to peaks sequence reported by Rouba et al. [28] and Larin et al. [31]. The degree of separation between the two peaks, $A$ and $B$ is quantified using the resolution $R$ which is expressed by [32],

$$
R=\frac{\left(t_{r}\right)_{B}-\left(t_{r}\right)_{A}}{0.5\left(w_{B}+w_{A}\right)}
$$

where $t_{r}$ and $w$ is the retention time and baseline width (Full width at half maximum-FWHM) respectively. The resolution for all the peaks was calculated as presented in Table 2. A photoionization detector (PID) was installed at the exit of UV detection cell to validate the results. The signal obtained for the PID is shown in the inset of Figure 6. All the peaks of BTEX molecules measured with UV absorption are well-separated, and the corresponding resolution values are higher than those of PID. The peaks of ethylbenzene and xylene (-o) and xylenes $(m+p)$ are clearly demarcated from each other 
with peaks resolution values two times higher compared to the PID ones . This detection of BTEX mixture with separated peaks is attributed to the lower volume $(133.5 \mu \mathrm{L})$ of the PEEK cell with quick time response. For the case of PID, the gas mixture has to cross a porous membrane by diffusion.

Table 2. Peaks resolution calculated for BTEX using UV absorption spectrophotometer and PID.

\begin{tabular}{|c|c|c|c|c|c|c|c|}
\hline \multirow{3}{*}{$\begin{array}{c}\text { Peaks } \\
\text { Resolution }\end{array}$} & Detector & Benzene & Toluene & Etl & & $\begin{array}{l}\text { Xylene } \\
(-m,-p)\end{array}$ & $\begin{array}{c}\text { Xylene } \\
(-0)\end{array}$ \\
\hline & $\begin{array}{c}\text { UV absorption } \\
\text { spectromphotometer }\end{array}$ & \multicolumn{2}{|c|}{10.44} & 12.49 & \multicolumn{2}{|c|}{2.35} & 6.21 \\
\hline & PID & \multicolumn{2}{|c|}{4.28} & 7.69 & & & 1.50 \\
\hline
\end{tabular}

The response of the detector was tested for total BTEX concentration of $60 \mathrm{ppm}, 30 \mathrm{ppm}$ and $15 \mathrm{ppm}$ with each BTEX molecule of concentration of $10 \mathrm{ppm}, 5 \mathrm{ppm}$ and $2.5 \mathrm{ppm}$, respectively. The lower concentration (i.e. $5 \mathrm{ppm}$ and $2.5 \mathrm{ppm}$ ) were generated by diluting the mixture of BTEX (10 ppm) with $\mathrm{N}_{2}$ at a ratio of 1:2 and 1:4 respectively with a setup given in Figure S1 (supplementary file). For the case of 5 ppm BTEX mixture, all the BTEX molecules were detected. On the other hand, for $2.5 \mathrm{ppm}$ BTEX mixture, xylenes and toluene were detected while benzene and ethylbenzene were not clearly detected on the chromatogram due to their lower values of the absorption coefficient. The sensitivity (peak area under the aborbance curve) for $10 \mathrm{ppm}, 5 \mathrm{ppm}$ and 2.5ppm are summarized in Figure 7. A good linearity was obtained for toluene, xylenes $(m+p)$ and xylene $(o)$ as shown in calibration curve in Figure 8 .

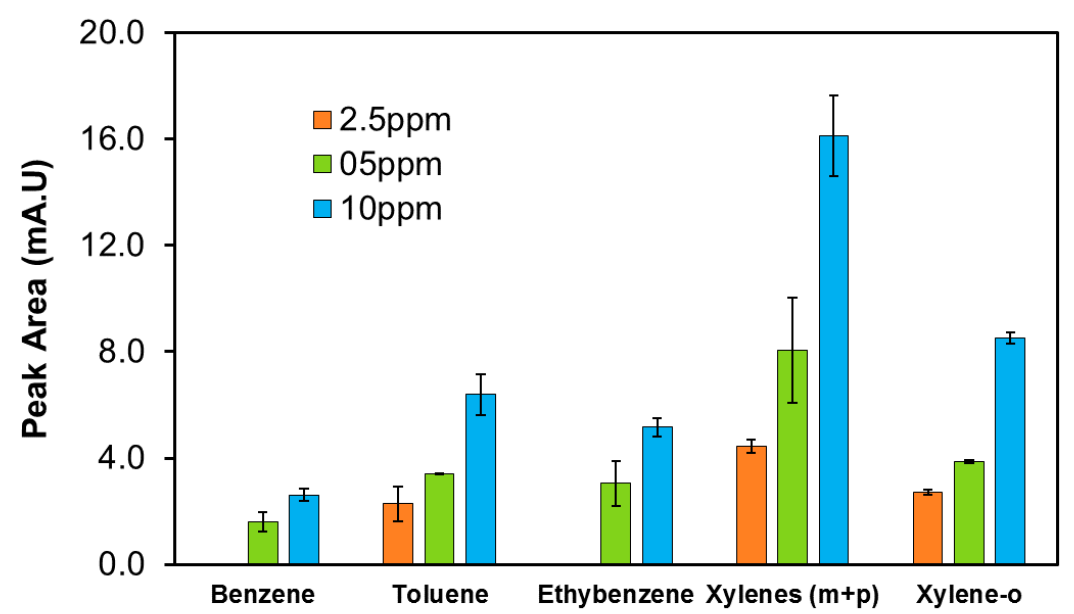

Figure 7. Absorbance for each BTEX component using BTEX concentration in the range 2.5-10 ppm with a $50 \mathrm{~cm}$ PEEK cell at a column temperature of $60^{\circ} \mathrm{c}$. 

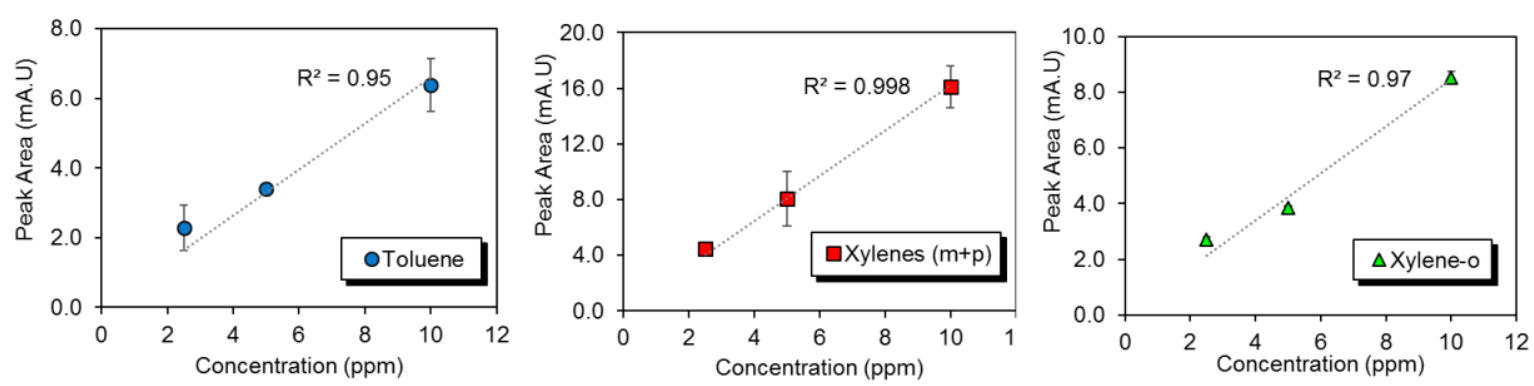

Figure 8. Calibration curve obtained from the chromatograms for toluene, xylenes $(m+p)$ and xylene-o.

The experiments were repeated 5 times for $10 \mathrm{ppm}$ with a $\mathrm{GC}$ column regulated at $60{ }^{\circ} \mathrm{C}$ and the absorbance spectra obtained are shown in Figure S2. The RSD values for the peaks were calculated for each molecule and a good repeatability was obtained for all BTEX molecules (RSD <10\%) at $10 \mathrm{ppm}$ of BTEX mixture, the RSD ranging between few percent and $27 \%$ for $5 \mathrm{ppm}$. The peaks resolution for the five chromatograms was calculated using Equation 2. As shown in Figure 9, the peak resolution values are lower than $10 \%$ for all the BTEX components. All the BTEX component species therefore, exhibit a good repeatability. Ethylbenzene shows relative higher RSD values due to its lower absorbance coefficient and closer proximity to peak of xylenes $(m+p)$.

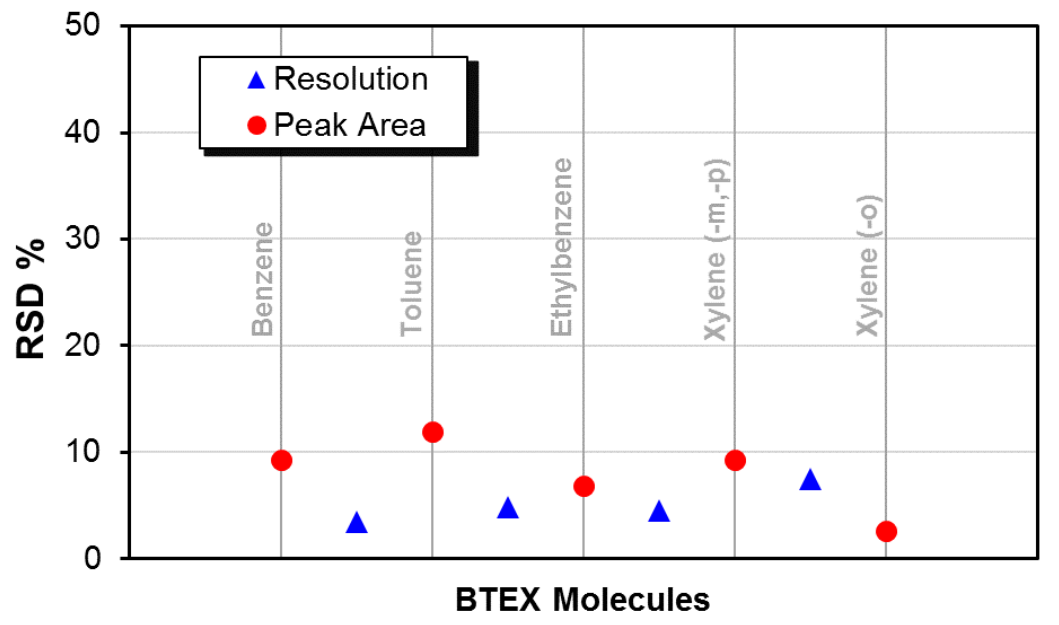

Figure 9. Relative Standard Deviation (RSD\%) for peaks area of BTEX molecule and resolution calculated for each consecutive pair of BTEX molecule.

\section{Conclusions}

In this work, we have developed a novel deep-UV absorption spectrophotometer using a lowvolume $(133.5 \mu \mathrm{L})$ PEEK gas cell coupled with a LED and PMT for the detection of air-borne BTEX.

287 The spectrophotometer was tested with different lengths of the gas cell for detection of toluene mixtures.

288 A sensitivity of $107.1 \mu \mathrm{AU} / \mathrm{ppm}$ with LOD of $3.5 \mathrm{ppm}$ was obtained for a gas cell of $30 \mathrm{~cm}$. The prototype was then tested with a $\mu \mathrm{GC}$, and all the separated BTEX components in the $\mu \mathrm{GC}$ column 
290 were detected with a separate peak for each species. It demonstrated better performance for the detection 291 of BTEX peaks with higher value of peak resolution compared to the commercial detector used. The 292 setup exhibited good repeatability at $10 \mathrm{ppm}(\mathrm{RSD}<10 \%)$ for the absorbance and retention time or peaks 293 resolution measurement for BTEX mixture. The proposed design offers a simple, cost-effective and 294 easy-to-made approach for the application of portable deep-UV absorption spectrophotometry, the cost 295 of the proposed design being approximately half of the conventional lamp-based system requiring based 296 on deuterium lamp and spectrometer. The proposed setup with low-volume cell has the potential to be 297 extended for the chromatographic studies of analytes in liquid media by replacing the flow injection 298 setup and the LED according to the UV-visible stectra of the target species. 
This work was funded by European Union's Horizon 2020 research and innovation programme under the Marie Skłodowska-Curie Innovative Training Network-MIGRATE (Miniaturized Gas flow 303 foR Applications with Enhanced Thermal Effects), grant agreement No. 643095 [H2020-MSCA-ITN304 2014]. This work has also received funding from the Clean Sky 2 Joint Undertaking under the European 305 Union's Horizon 2020 research and innovation program under grant agreement No 687014 (MACAO 306 Project).

307

308 


\section{Highlights}

- A novel deep-UV absorption spectrophotometer using a low-volume (133.5 $\mu \mathrm{L})$ PEEK gas cell was developed for the detection and identification of air-borne BTEX.

- The prototype is based on using potable UV emission source and UV photodetector.

- A limit of detection of $1.41 \mathrm{ppm}$ was obtained for the direct injection of toluene.

- The detector was tested with a micro gas-chromatography $(\mu \mathrm{GC})$ for the detection and identifcations of BTEX mixture in nitrogen carrier gas and all the molecules were separated and detected.

- The design offer a low-cost and easy to fabricate setup for spectrophotometric analysis on portable platform and can extended to be used in gas or liquid media. 


\section{References}

[1] R. Koppmann, "Chemistry of Volatile Organic Compounds in the Atmosphere," in Handbook of Hydrocarbon and Lipid Microbiology, Berlin, Heidelberg: Springer Berlin Heidelberg, 2010, pp. 267-277.

[2] B. Noziere et al., "The Molecular Identification of Organic Compounds in the Atmosphere: State of the Art and Challenges," Chem. Rev., vol. 115, no. 10, pp. 3919-3983, 2015.

[3] P. Schneider et al., "Indoor and outdoor BTX levels in German cities," Sci. Total Environ., vol. 267, no. 1-3, pp. 41-51, Feb. 2001.

[4] L. A. Wallace, "Major sources of benzene exposure," Environ. Health Perspect., vol. 82, pp. $165-169,1989$.

[5] G. A. Ayoko, "Volatile Organic Compounds in Indoor Environments," in Environmental Chemistry, vol. 4, 2004, pp. 1-35.

[6] K. Kawamura, M. Vestergaard, M. Ishiyama, N. Nagatani, T. Hashiba, and E. Tamiya, "Development of a novel hand-held toluene gas sensor: Possible use in the prevention and control of sick building syndrome," Measurement, vol. 39, no. 6, pp. 490-496, Jul. 2006.

[7] D. Pyatt and S. Hays, "A review of the potential association between childhood leukemia and benzene," Chemico-Biological Interactions, vol. 184, no. 1-2. pp. 151-164, 19-Mar-2010.

[8] WHO, "Exposure to Benzene: A Major public health concern," 2010.

[9] "Benzene (IARC Summary \&amp; Evaluation, Supplement7, 1987)." [Online]. Available: http://www.inchem.org/documents/iarc/suppl7/benzene.html. [Accessed: 26-Mar-2017].

[10] P. Patnaik, A comprehensive guide to the hazardous properties of chemical substances. John Wiley, 2007.

[11] R. Kandyala, S. P. Raghavendra, and S. Rajasekharan, "Xylene: An overview of its health hazards and preventive measures," J. Oral Maxillofac. Pathol., vol. 14, no. 1, p. 1, Jan. 2010.

[12] M. . Barson, "NIOSH-Pocket Guide to Chemical Hazards," Pittsburgh USA, 2005.

[13] A. Mirzaei, J. H. Kim, H. W. Kim, and S. S. Kim, "Resistive-based gas sensors for detection of benzene, toluene and xylene (BTX) gases: A review," Royal Society of Chemistry, 2018.

[14] X. Zhou, S. Lee, Z. Xu, and J. Yoon, "Recent Progress on the Development of Chemosensors for Gases," Chemical Reviews, vol. 115, no. 15. pp. 7944-8000, 2015.

[15] G. Coelho Rezende, S. Le Calvé, J. J. Brandner, and D. Newport, "Micro photoionization detectors," Sensors and Actuators, B: Chemical, vol. 287. Elsevier, pp. 86-94, 15-May-2019.

[16] J. Devkota, P. Ohodnicki, and D. Greve, "SAW Sensors for Chemical Vapors and Gases," Sensors, vol. 17, no. 4, p. 801, Apr. 2017.

[17] C. McDonagh, C. S. Burke, and B. D. MacCraith, "Optical chemical sensors," Chem. Rev., vol. 108 , no. 2, pp. 400-422, 2008.

[18] S. Khan, S. Le Calvé, and D. Newport, "A review of optical interferometry techniques for VOC detection," Sensors and Actuators, A: Physical, vol. 302. Elsevier B.V., p. 111782, 01-Feb-2020.

[19] J. Mikolajczyk, "Detection of gaseous compounds with different techniques," Metrol. Meas. Syst., vol. 23, no. 2, pp. 202-224, 2016.

[20] J. Hodgkinson and R. P. Tatam, "Optical gas sensing: a review," Meas. Sci. Technol., vol. 24, no. 1, p. 012004, 2013.

[21] S. Khan, D. Newport, and S. Le Calvé, "Gas Detection Using Portable Deep-UV Absorption Spectrophotometry: A Review," Sensors, vol. 19, no. 5210, 2019.

[22] F. Pena-Pereira, I. Costas-Mora, V. Romero, I. Lavilla, and C. Bendicho, "Advances in miniaturized UV-Vis spectrometric systems," TrAC - Trends Anal. Chem., vol. 30, no. 10, pp. 1637-1648, 2011.

[23] H. S. Eckhardt, K. F. Klein, B. Spangenberg, T. Sun, and K. T. V. Grattan, "Fibre-optic UV systems for gas and vapour analysis," J. Phys. Conf. Ser., vol. 85, no. 1, 2007.

[24] R. Kötschau, H. S. Eckhardt, K. Klein, K. Behler, and G. Hillrichs, "Gas-Analysis in the UVregion using a long-length Hollow-Core-Waveguide," in Optical Fibers and Sensors for Medical Applications III, Proceedings of SPIE, 2003, vol. 4957, pp. 134-141.

[25] D. A. Bui and P. C. Hauser, "A deep-UV light-emitting diode-based absorption detector for benzene, toluene, ethylbenzene, and the xylene compounds," Sensors Actuators, B Chem., vol. 235, pp. 622-626, 2016. 
[26] P. A. Schweitzer, Mechanical and Corrosion-Resistant Properties of Plastics and Elastomers. CRC PRESS, 2000.

[27] I. Lara-lbeas et al., "BTEX near real-time monitoring in two primary schools in La Rochelle, France," Air Qual. Atmos. Heal., vol. 11, no. 9, pp. 1091-1107, Nov. 2018.

[28] R. Nasreddine, V. Person, C. A. Serra, and S. Le Calvé, "Development of a novel portable miniaturized GC for near real-time low level detection of BTEX," Sensors Actuators B Chem., vol. 224, pp. 159-169, 2016.

[29] F. Menges, "Spectragryph - optical spectroscopy software"." 2019.

[30] I. E. Gordon et al., "The HITRAN2016 molecular spectroscopic database," J. Quant. Spectrosc. Radiat. Transf., vol. 203, pp. 3-69, Dec. 2017.

[31] A. Larin, P. C. Womble, and V. Dobrokhotov, "Novel highly-integrated mems based solid state detectors for analytical gas chromatography," Sensors Actuators, B Chem., vol. 256, pp. 10571068, Mar. 2018.

[32] D. Harvey, "Modern Analytical Chemistry," Anal. Chem., vol. 34, no. 7, p. 798, 1962. 